\title{
Diffraction by a plane angular sector, a new derivation
}

\author{
Hansen, Thokild B.
}

Published in:

I E E E Transactions on Antennas and Propagation

Link to article, DOI:

10.1109/8.102757

Publication date:

1990

Document Version

Publisher's PDF, also known as Version of record

Link back to DTU Orbit

Citation (APA):

Hansen, T. B. (1990). Diffraction by a plane angular sector, a new derivation. I E E E Transactions on Antennas and Propagation, 38(11), 1892-1894. https://doi.org/10.1109/8.102757

\section{General rights}

Copyright and moral rights for the publications made accessible in the public portal are retained by the authors and/or other copyright owners and it is a condition of accessing publications that users recognise and abide by the legal requirements associated with these rights.

- Users may download and print one copy of any publication from the public portal for the purpose of private study or research.

- You may not further distribute the material or use it for any profit-making activity or commercial gain

- You may freely distribute the URL identifying the publication in the public portal

If you believe that this document breaches copyright please contact us providing details, and we will remove access to the work immediately and investigate your claim. 
Thus, other than the null series result, the current in the shadow region does not have an asymptotic series representation. This is an important result because it is the first time (to the author's knowledge) that the failure of an $\mathrm{L}-\mathrm{K}$ series in the shadow region has been both demonstrated and explained.

While the $\mathbf{L}-\mathrm{K}$ series does not lead to an exact result for the surface current density, it still holds the potential for providing a tractable improvement to a pure geometrical optics solution. This fact is demonstrated by Ansorge's [5] calculations for scattering by a dielectric sphere using the ray optics field approach. The attractiveness of the current approach as developed here is due in large part to the fact that the complete $\mathbf{L}-\mathbf{K}$ representation can be developed entirely from an integral of known functions, i.e.,

$$
\int \nabla_{0} G(|\Delta \vec{r}|) \exp \left(j \vec{k}_{i} \cdot \Delta \vec{r}\right) d \vec{r}_{t_{0}}
$$

While a complete $\mathbf{L}-\mathbf{K}$ series development for this integral is prohibitive, it may be possible to obtain the terms up to and including $k_{0}^{-2}$ [7]. One of the primary advantages of obtaining the $k_{0}^{-1}$ and $k_{0}^{-2}$ corrections to the $k_{0}^{0}$ asymptotic expansion of this integral is the recovery of some of the cross-polarizing properties of a rough surface in the high frequency (but not optical) limit.

The results obtained in this communication raise an interesting point. Usually, the first frequency dependent correction to physical optics comes from the nonzero width of the transition zone between the illuminated and shadowed regions on the scatterer and the propagation of creeping waves into the shadow zone. If the $\mathrm{L}-\mathrm{K}$ series produces a result that is identically zero in the shadow zone, what is the physical source of the $k_{0}^{-n}, n=1,2, \cdots$ terms in the illuminated zone? It would appear that this may be due to the transition zone encroaching into the illuminated zone as the frequency is decreased. This is the kind of question that needs to be answered if there is to be a full understanding of the Luneburg-Kline asymptotic representation.

It should be noted that $\vec{s}_{1}(\vec{r})$ in $(21 \mathrm{~b})$ has essentially been computed by Chaloupka and Meckelburg [8] in a very clever application of integration by parts. Their results should provide the basic ingredients to study the question raised in the previous paragraph.

\section{ACKNOWLEDGMENT}

The author is indebted to Drs. Werner Kohler and Amr Shaarawi for many helpful discussions on this topics.

\section{REFERENCES}

[1] V. A. Fock, Electromagnetic Diffraction and Propagation Problems, Elmsford, NY: Pergamon, 1965.

[2] J. J. Bowman, T. B. A. Senior, and P. L. E. Uslenghi, Electromagnetic and Acoustic Scattering by Simple Shapes. Amsterdam: North-Holland, 1969.

[3] S. W. Lee, "Electromagnetic reflection from a conducting surface: geometrical optics solution," IEEE Trans. Antennas Propagat., vol. AP-23, pp. 184-191, 1975.

[4] C. E. Schensted, "Electromagnetic and acoustical scattering by a semi-infinite body of revolution," J. Appl. Phys., vol. 26, pp. $306-308,1955$

[5] H. Ansorge, "Electromagnetic reflection from a curved dielectric interface," IEEE Trans. Antennas Propagat., vol. AP-34, pp. $842-845,1986$.

[6] G. S. Brown, "The validity of shadowing corrections in rough surface scattering," Radio Sci., vol. 19, pp. 1461-1468, 1984.

[7] N. Chako, "Asymptotic expansions of double and multiple integrals occurring in diffraction theory," J. Inst. Math. Appl., vol. 1, pp. $372-422,1965$.
[8] H. Chaloupka and H.-J. Meckleburg, "Improved high frequency current approximation for curved conducting surfaces," $A E U$, vol. 39, pp. $245-250,1985$.

\section{Diffraction by a Plane Angular Sector, A New Derivation}

\author{
THORKILD B. HANSEN
}

Abstract-The purpose of the present communication is to present an alternative derivation of the exact solution to the scattering problem in which a Hertz dipole illuminates a perfectly conducting plane angular sector.

\section{INTRODUCTION}

The geometrical theory of diffraction (GTD) is one of the most widely used methods for calculating scattered electromagnetic fields. In this theory, the scattered field is approximated by a series in which each term is a contribution from a certain point on the scatterer. These contributing points on the scatterer are called critical points by Van Kampen [1]. To calculate such contributions, diffraction coefficients belonging to each critical point are needed. Diffraction coefficients are found from exact solutions to so-called canonical scattering problems. As an example, the edge diffraction coefficient for the perfectly conducting edge is found from the exact solution to the infinite perfectly conducting wedge. Similarly, the corner diffraction coefficient may be found in principle from the exact solution to a canonical scattering problem that contains a corner. Today there exists only one such solution, that of diffraction from a perfectly conducting plane angular sector, although a corner diffraction coefficient has not been found from it. However, progress has recently been made by Smyshlyaev [2], [3] in deriving a corner diffraction coefficient from this solution.

A plane angular sector is a section of a plane bounded by two half-lines which have common endpoints. As an example, the plane angular sector is a quarter-plane if the half-lines are orthogonal to each other. The vector solution to this scattering problem was first found by Satterwhite [4]. This solution has been used for numerical calculations in several papers e.g. [5]-[7]. Corrections to some misprints in the vector wave function $\bar{N}$ appearing in these references are given in Section III. Because Satterwhite's solution is the only one existing for a scattering problem where the scatterer contains a corner, it would be valuable to verify it. The purpose of this communication is to present a new derivation of Satterwhite's solution. No attempt is made to derive a corner diffraction coefficient from it.

The method used by Satterwhite [4] can be described as follows. The total electric field is written as a series expansion of vector wave functions. The expansion coefficients are found by integration over a surface which is a sphere, with center at the tip of the plane angular sector, except for an infinitesimal cut around the plane angular sector. During this integration, use is made of Green's second identity for vectors, the divergence theorem, and orthogonal-

Manuscript received August 8, 1989; revised March 12, 1990.

The author is with the Electromagnetics Institute, The Technical University of Denmark, DK-2800 Lyngby, Denmark.

IEEE Log Number 9038597. 


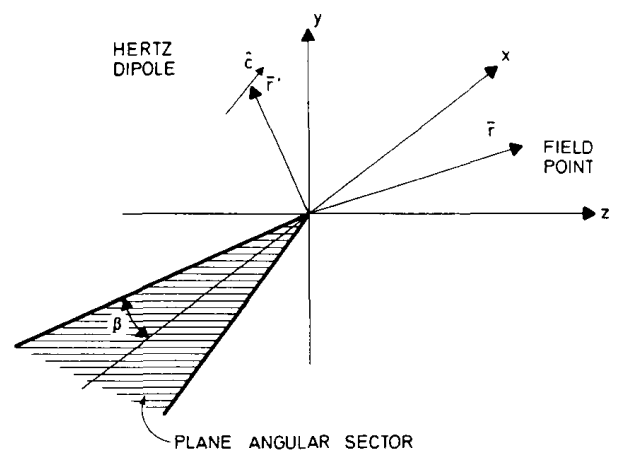

Fig. 1. Physical configuration.

ity relations for Lamé functions. From this expansion of the total electric field the electric dyadic Green's function for the perfectly conducting plane angular sector is identified.

In this communication, we use the Ohm-Rayleigh method [8] which is very different from that used by Satterwhite. To use the Ohm-Rayleigh method three orthogonality relations between vector wave functions are needed. These relations have been proved in [9]. For the sake of brevity, only one of them is proved in this communication. The proof is given in the Appendix where all three orthogonality relations are listed. The agreement of the solutions obtained here with that obtained by Satterwhite demonstrates that the solution and the orthogonality relations are correct.

\section{Formulation of the Problem}

The problem considered in this communication is shown in Fig. 1. A Hertz dipole with current moment $\bar{c}$, located at the point $\bar{r}^{\prime}$, is illuminating a perfectly electrically conducting plane angular sector. The plane angular sector is situated symmetrically about the negative $x$-axis in the plane $y=0$. The parameter $\beta$ denotes the angle between the edges of the plane angular sector and the negative $x$-axis. We let $\beta \in] 0, \pi / 2[$. In the following, the point set that forms the plane angular sector is denoted by PAS.

The electric field $\bar{E}(\bar{r})$ at the field point $\bar{r}$ can be written as [8]

$$
\bar{E}(\bar{r})=j \omega \mu \overline{\bar{G}}\left(\bar{r} \mid \bar{r}^{\prime}\right) \cdot \bar{c}
$$

where $\mu$ is the permeability, $\omega$ the frequency (time dependence $e^{j \omega t}$ is assumed), and $\overline{\bar{G}}(\bar{r}, \bar{r})$ the electric dyadic Green's function.

The dyadic Green's function satisfies the following equations:

$$
\begin{aligned}
& \nabla \times \nabla \times \overline{\bar{G}}-\kappa^{2} \overline{\bar{G}}=-\overline{\bar{I}} \delta\left(\bar{r}-\bar{r}^{\prime}\right) \\
& \hat{y} \times \overline{\bar{G}}\left(\bar{r} \mid \bar{r}^{\prime}\right)=0, \quad \text { when } \bar{r} \in \text { PAS }
\end{aligned}
$$

where $\overline{\bar{I}}$ denotes the identity dyad and $\kappa$ is the propagation constant. Furthermore, $\overline{\bar{G}}\left(\bar{r} \mid \bar{r}^{\prime}\right)$ must satisfy the edge condition, the corner condition, and the radiation condition. The edge and corner conditions ensure that the field energy at the edges and corner is finite.

\section{Determination of the Dyadic Green's Function}

In order to apply the method of separation of variables to the problem of scattering from a plane angular sector we must find a coordinate system in which:

1) the vector-wave equation is separable;

2) the plane angular sector is formed by one or more of the coordinate surfaces.

From Morse and Feshbach [10] we see that there are only six coordinate systems satisfying 1) and that the spheroconal coordinate system $(r, \theta, \phi)$ is the only one of these satisfying 2$)$.
The plane angular sector shown in Fig. 1 is given by the equation $\theta=\pi$ if the ellipticity parameter $k$ associated with the coordinate system is equal to $\cos \beta$. For a detailed discussion of the spheroconal coordinate system, see [9] or [11]. By inserting $\Psi=$ $R(r) \Theta(\theta) \Phi(\phi)$ in the scalar Helmholtz equation

$$
\nabla^{2} \Psi+\alpha^{2} \Psi=0, \quad \alpha \in R_{+}
$$

performing the separation with two separation constants denoted by $\mu$ and $v(v+1)$, one finds that the radial functions $R(r)$ are spherical Bessel functions and that the angular functions $\Theta(\theta)$ and $\Phi(\phi)$ are Lamé functions. The following notation for the scalar wave functions $\Psi(\bar{r}, \alpha)$ is useful.

$$
\Psi_{s m i}^{A}(\bar{r}, \alpha), \quad A \in\{I, I I\}, \quad s \in\{e, o\}, m \in N \text { and } i \in\{1,2\}
$$

where $A=I$ when $R(r)$ is a spherical Bessel function of the first kind and $A=\mathrm{II}$ when $R(r)$ is a spherical Hankel function of the second kind. Index $s=e$ when $\Theta(\theta)$ and $\Phi(\phi)$ are even, and $s=0$ when $\Theta(\theta)$ and $\Phi(\phi)$ are odd. Index $i$ is equal to one or two when $\Psi$ satisfies the Direchlet or Neumann condition on the plane angular sector, respectively. The eigenvalue pairs $(\mu, v)$ belonging to each of the values of $(i, s)$ are numbered, and $\left(\mu_{s m i}, v_{s m i}\right)$ denotes the $m$ th of these. The corresponding subscript $m$ on $\Psi_{s m i}$ implies that $\Psi_{s m i}$ is the eigenfunction belonging to this eigenvalue pair.

Vector wave functions satisfying the vector Helmholtz equation are constructed in the following way:

$$
\bar{M}_{s m i}^{A}(\bar{r}, \alpha)=\nabla \times\left(\bar{r} \Psi_{s m i}^{A}(\bar{r}, \alpha)\right)
$$

and

$$
\bar{N}_{s m i}^{A}(\bar{r}, \alpha)=\frac{1}{\alpha} \nabla \times \nabla \times\left(\bar{r} \Psi_{s m i}^{A}(\bar{r}, \alpha)\right) .
$$

The correct form of $\bar{N}_{s m i}(\bar{r}, \alpha)$ is

$$
\begin{aligned}
\bar{N}_{s m i}^{A}= & v_{s m i}\left(v_{s m i}+1\right) \frac{R_{s m i}^{A}(\alpha r)}{\alpha r} \Theta_{s m i}(\theta) \Phi_{s m i}(\phi) \hat{r} \\
& +\frac{\frac{d}{d r}\left[r R_{s m i}^{A}(\alpha r)\right]}{\alpha r \sqrt{k^{2} \sin ^{2} \theta+k^{\prime 2} \sin ^{2} \phi}} \\
& \cdot\left[\sqrt{1-k^{\prime 2} \cos ^{2} \phi} \Theta_{s m i}(\theta) \Phi_{s m i}^{\prime}(\phi) \hat{\phi}\right. \\
& \left.+\sqrt{1-k^{2} \cos ^{2} \theta} \Theta_{s m i}^{\prime}(\theta) \Phi_{s m i}(\phi) \hat{\theta}\right]
\end{aligned}
$$

$\underline{N}_{s m i}$ riting down the explicit expressions for $\bar{M}_{s m i}(\bar{r}, \alpha)$ and $\bar{N}_{s m i}(\bar{r}, \alpha)$ one finds that only $\bar{M}_{s m 2}(\bar{r}, \alpha)$ and $\bar{N}_{s m 1}(\bar{r}, \alpha)$ have zero tangential component on the plane angular sector. We can therefore write the electric dyadic Green's function as

$$
\begin{aligned}
\overline{\bar{G}}\left(\bar{r} \mid \bar{r}^{\prime}\right)=\int_{0}^{+\infty}\left[\sum_{s, m} \bar{M}_{s m 2}^{A}(\bar{r}, \alpha) \bar{A}_{s m}\left(\bar{r}^{\prime}, \alpha\right)\right. & \\
& \left.+\bar{N}_{s m 1}^{A}(\bar{r}, \alpha) \bar{B}_{s m}\left(\bar{r}^{\prime}, \alpha\right)\right] d \alpha .
\end{aligned}
$$

By imposing the finite-energy corner condition one finds that $A=I$. By inserting (4) into (2) and using that the vector wave functions satisfy the vector Helmholtz equation one obtains

$$
\begin{aligned}
\int_{0}^{+\infty}\left[\left(\alpha^{2}-\kappa^{2}\right) \sum_{s, m} \bar{M}_{s m 2}^{I}(\bar{r}, \alpha) \bar{A}_{s m}\left(\bar{r}^{\prime}, \alpha\right)\right. \\
\left.\quad+\bar{N}_{s m 1}^{I}(\bar{r}, \alpha) \bar{B}_{s m}\left(\bar{r}^{\prime}, \alpha\right)\right] d \alpha=-\overline{\bar{I}} \delta\left(\bar{r}-\bar{r}^{\prime}\right)
\end{aligned}
$$

Using the orthogonality relations (9)-(11) the following expressions 
for the expansion coefficients $\bar{A}_{s m}$ and $\bar{B}_{s m}$ are obtained

$$
\bar{A}_{s m}\left(\bar{r}^{\prime}, \alpha\right)=-\frac{2}{\pi} \frac{\alpha^{2}}{\alpha^{2}-\kappa^{2}} \frac{1}{\Lambda_{s m 2}} \bar{M}_{s m 2}^{I}\left(\bar{r}^{\prime}, \alpha\right)
$$

and

$$
\bar{B}_{s m}\left(\bar{r}^{\prime}, \alpha\right)=-\frac{2}{\pi} \frac{\alpha^{2}}{\alpha^{2}-\kappa^{2}} \frac{1}{\Lambda_{s m 1}} \bar{N}_{s m 1}^{I}\left(\bar{r}^{\prime}, \alpha\right) .
$$

Insertion of (6) and (7) into (4) and integration with respect to $\alpha$, [9] gives our final expressions for the electric dyadic Green's function:

$$
\begin{aligned}
\overline{\bar{G}}\left(\bar{r} \mid \bar{r}^{\prime}\right) & =j \kappa \sum_{s, m}\left[\bar{M}_{s m 2}^{I I}(\bar{r}, \kappa) \bar{M}_{s m 2}^{I}\left(\bar{r}^{\prime}, \kappa\right) \frac{1}{\Lambda_{s m 2}}\right. \\
& \left.+\bar{N}_{s m 1}^{I I}(\bar{r}, \kappa) \bar{N}_{s m 1}^{I}\left(\bar{r}^{\prime}, \kappa\right) \frac{1}{\Lambda_{s m 1}}\right], \quad \text { when }|\bar{r}|>\left|\bar{r}^{\prime}\right|
\end{aligned}
$$

and the same expression with $\bar{r}$ and $\bar{r}^{\prime}$ interchanged when $|\bar{r}|<$ $\left|\bar{r}^{\prime}\right|$.

This solution, which agrees with that in [4], is shown in [4] and [9] to satisfy the radiation and the edge condition. Explicit expressions for $\bar{M}_{s m i}$ and $\bar{N}_{s m i}$ may be found in [4] and [9].

Note that when fields are to be calculated within the source region an additional term must be added to the expression in (8) [12], [13].

In conclusion, the solution obtained by Satterwhite [4] is critically confirmed by using the Ohm-Rayleigh method of solution to determine the electric dyadic Green's function for the perfectly conducting plane angular sector.

\section{APPENDIX}

\section{ORTHOGONALITY RELATIONS}

The formulas shown in this Appendix are proved in [9] and, to the author's knowledge, have not previously been published.

$$
\begin{gathered}
\int_{R^{3}} \bar{M}_{s m 2}^{I}(\bar{r}, \alpha) \cdot \bar{N}_{s^{\prime} m^{\prime} 1}^{I}\left(\bar{r}, \alpha^{\prime}\right) d V=0 \\
\int_{R^{3}} \bar{M}_{s m 2}^{I}(\bar{r}, \alpha) \cdot \bar{M}_{s^{\prime} m^{\prime} 2}^{I}\left(\bar{r}, \alpha^{\prime}\right) d V \\
=\Lambda_{s m 2} \frac{\pi}{2} \frac{\delta\left(\alpha-\alpha^{\prime}\right)}{\alpha^{2}} \delta_{s s^{\prime}} \delta_{m m^{\prime}} \\
\int_{R^{3}} \bar{N}_{s m 1}^{I}(\bar{r}, \alpha) \cdot \bar{N}_{s^{\prime} m^{\prime} 1}^{I}\left(\bar{r}, \alpha^{\prime}\right) d V \\
=\Lambda_{s m 1} \frac{\pi}{2} \frac{\delta\left(\alpha-\alpha^{\prime}\right)}{\alpha^{2}} \delta_{s s^{\prime}} \delta_{m m^{\prime}}
\end{gathered}
$$

where $\Lambda_{s m 1}$ are normalization constants and $\delta_{p q}=0$ when $p \neq q$ and $\delta_{p p}=1$.

$$
\text { Proof of (10) }
$$

The integral in (10) can be written as

$$
\begin{aligned}
\int_{0}^{+\infty} j_{v_{s m 2}}(\alpha r) j_{v_{s^{\prime} m^{\prime} 2}}\left(\alpha^{\prime} r\right) r^{2} d r \int_{0}^{\pi} \int_{-\pi}^{\pi} \bar{m}_{s m 2}(\theta, \phi) \\
\cdot \bar{m}_{s^{\prime} m^{\prime} 2}(\theta, \phi) \sigma(\theta, \phi) d \phi d \theta
\end{aligned}
$$

where $j_{v}(z)$ is the spherical Bessel function and $\bar{m}_{s m 2}(\theta, \phi)$ is the angular dependent part of the vector wave function $\bar{M}_{s m 2}(\bar{r}, \alpha)$.
Furthermore, $h_{1} h_{2} h_{3}=r^{2} \sigma(\theta, \phi)$ where $h_{1}, h_{2}$, and $h_{3}$ are the metrical coefficients in the spheroconal coordinate system [11]. Note that the double integral is independent of $\alpha, \alpha^{\prime}$, and $r$. In order to evaluate the double integral in (12) we note that

$$
\begin{aligned}
\nabla \cdot\left(\Psi_{s m 2}(\bar{r}, \alpha) \nabla \Psi_{s^{\prime} m^{\prime} 2}(\bar{r}, \alpha)\right) & =-\alpha^{2} \Psi_{s m 2}(\bar{r}, \alpha) \Psi_{s^{\prime} m^{\prime} s}(\bar{r}, \alpha) \\
& +\nabla \Psi_{s m 2}(\bar{r}, \alpha) \cdot \nabla \Psi_{s^{\prime} m^{\prime} 2}(\bar{r}, \alpha) .
\end{aligned}
$$

By integrating this identity over a spherical volume with center at $(0,0,0)$, using the divergence theorem and the orthogonality relations for Lamé functions [11, appendix II], one finds that the double integral in (12) is zero when $s \neq s^{\prime}$ or $m \neq m^{\prime}$. By using $[8$, p. 11 , eq. (26)] and denoting the double integral in (12) by $\Lambda_{s m 2}$ when $s=s^{\prime}$ and $m=m^{\prime}$, the formula (10) is proved.

\section{REFERENCES}

[1] N. G. Van Kampen, "An asymptotic treatment of diffraction problems," Physica, vol. 14, pp. 575-589, Jan. 1949.

[2] V. P. Smyshlyaev, "On the diffraction of waves by cones at high frequencies," LOMI, preprint E-9-89, Leningrad, 1989.

[3] _-, "Diffraction of electromagnetic waves by conical obstacles in high frequencies," in Proc. Int. URSI Symp. Electromagn. Wave Theory, Stockholm, Sweden, Aug. 1989.

[4] R. S. Satterwhite, "Diffraction by a plane angular sector," Ph.D. dissertation, Ohio State Univ., Columbus, OH, 1969.

[5] J. N. Sahalos and G. A. Thiele, "The eigenfunction solution for scattered fields and surface currents of a vertex," IEEE Trans. Antennas Propagat., vol. AP-31, pp. 206-221, Jan. 1983.

[6] E. Vafiadis and J. N. Sahlos, "Diffraction by a perfectly conducting elliptic cone," Archiv für Elektrotechnik, vol. 66, pp. 117-124, 1983.

[7] R. S. Satterwhite, "Diffraction by a quarter plane, the exact solution, and some numerical results," IEEE Trans. Antennas Propagat., vol. AP-22, pp. 500-503, May 1974.

[8] C. Tai, Dyadic Green's Function in Electromagnetic Theory. New York: Intext, 1971.

[9] T. B. Hansen, "Corner diffraction," M.Sc. thesis. Electromagn. Inst., Tech. Univ. Denmark, 1988 (in Danish).

[10] P. M. Morse and H. Feshbach, Methods of Theoretical Physics. New York: McGraw-Hill, 1953, pp. 655-665, 1767.

[11] L. Kraus and L. Levine, "Diffraction by an elliptic cone," Commun. Pure Appl. Math., vol. XIV, pp. 49-68, 1961.

[12] P. H. Pathak, "On the eigenfunction expansion of electromagnetic dyadic Green's functions," IEEE Trans. Antennas Propagat., vol. AP-31, pp. 837-846, Nov. 1983.

[13] A. D. Yaghjian, "Electric dyadic Green's functions in the source region," Proc. IEEE, vol. 68, no. 2, pp. 248-263, Feb. 1980.

\section{The Electromagnetic Field of a Slotted Elliptic Cone}

$$
\text { E. VAFIADIS, MEMBER, IEEE, AND J. N. SAHALOS, SENIOR }
$$
MEMBER, JFEE

Abstract-The exact solution for the electromagnetic field diffracted by a perfectly conducting slotted elliptic cone is determined. The solution is presented in the form of a dyadic Green's function, which is the most general form of a possible solution. Some numerical results are

Manuscript received September 27, 1989; revised May 30, 1990.

E. Vafiadis is with the Department of Electrical Engineering, University of Thrace, Xanthi, 67100 Greece.

J. N. Sahalos is with the Department of Physics, University of Thessaloniki, Thessaloniki, 54006 Greece.

IEEE Log Number 9038572. 